Military Technical College Kobry El-Kobbah, Cairo, Egypt

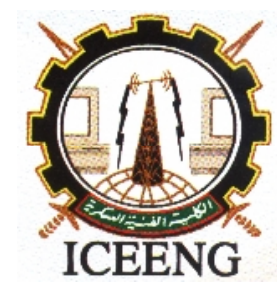

\author{
$8^{\text {th }}$ International Conference \\ on Electrical Engineering \\ ICEENG 2012
}

\title{
Selection of optimal filtration and prediction block in the single target tracking system with one imaging sensor under changeable daylight
}

\author{
By \\ Mikluc Davorin* Antonijević Goran* Andrić Milenko* Bondžulić Boban*
}

\section{$\underline{\text { Abstract: }}$}

The paper presents a system with one imaging sensor. Serial communication interface enables azimuth and elevation control, zoom setting, and changing the mode into infrared. Single target tracking in plane in multiple scenarios has been analysed in detail. By comparative analysis of CA models, Singer model and IMM algorithm with two models, and on the basis of root mean square error of position estimation, optimal filtration and prediction block has been selected. The first scenario shows linear target tracking at variable velocity. In the second scenario, the sensor tracks a maneuvering target. The third and fourth scenario present target tracking with infrared sensor with the cinematic characteristics identical to the first two scenarios. These are followed by scenarios in which the imaging and infrared sensors are controlled in target tracking, and the influence of different filtration and prediction blocks on the tracking is analysed. The last scenario shows target tracking under changeable conditions with automatic sensor control. All the scenario analyses are based on root mean square error of position.

\section{Keywords:}

Image restoration, remote sensing and image blur models 


\section{Introduction:}

Target tracking and detection is often based on sensor functioning in battlefield. Multifunctional sensor, i.e. platform, can be functional under different circumstances. However, that type of equipment is often robust. Hence the motive to analyse usage of simpler sensors and hardware which would be capable of meeting the needs of modern armament. A variety of lasers are used in civilian structures nowadays. Laser application in the armed forces is also rather frequent [1]. Laser precision makes many target tracking concepts possible. One example is the idea of synthesizing one target tracking system with one image sensor, [2], [3]. Object tracking is extremely important in security systems, fire control systems etc., [4]. Systems which use laser measurement (LMS) most frequently use Kalman filter in tracking laser glints, [5], [6]. This paper analyses laser glint tracking with different models of Kalman filter in order to select the optimal one, [7], [8]. The results can be used in different LMS systems, depending on minimising tracking error requirement and the processing time of systems. LMS systems include both laser glint tracking in visible spectrum and in infrared spectrum. The system suggested in this paper is the one consisting of a camera with three-mode option in different spectrums, visible, infrared and wide. The camera has pan and tilt option. Such a system can track targets under different circumstances, [9], [10], [11]. Simultaneously, system sensor can be easily transformed from a video sensor into the infrared one, [12].

Synthetic images were formed, [13], analysed and tracked through 50 Monte Carlo simulations. Video sequences were recorded in a scenario similar to synthetic images. An experiment in keeping with the mentioned scenarios was performed. All the results are shown in graphs with tracking trajectory and root mean square error criterion.

\section{System Description:}

Block scheme of one target tracking system is given in figure 1. Image sensor is Canon VC-C50i camera, with pan and tilt option. Communication between computer and camera is serial. Sensor output is a composite video signal. The sensor has the option of manageable infrared light cut filter implementation and manually implementable visible light cut filter. Constant velocity model (CV), constant acceleration model (CA), Singer model of Kalman filter and interactive multiple algorithm model (IMM) consisting of CV and CA models are used in filtration and prediction blocks. Image processing block pinpointed the target on the basis of gray treshold.

Camera characteristics are the following: resolution 720x576 pixels, composite output video signal, frame rate 25 frame/s, pan motion velocity is $90 \%$, tiltu motion velocity is $30^{\circ} \mathrm{s}$, includes manageable IC filter, adaptable field of vision option. Image processing block pinpoints the target on the basis of gray treshold. Pixel center is determined, and pixels are singled out from the background. Target coordinates are 
1 pixel.

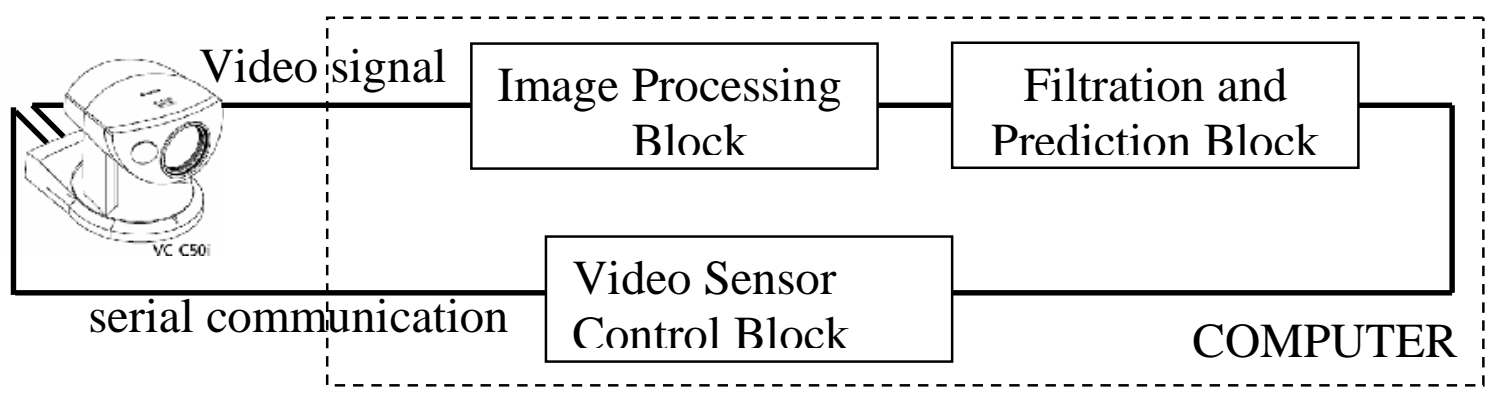

Figure (1): Video sensor target tracking block scheme

Sensor Monitoring Block transfers the information on camera motion via serial communication. Filtration and Prediction Block comprises Kalman filter models and performs target motion estimation. Selection of values for measurement noise and process noise variations. As the laser is handheld, there is a certain process noise, figure 2. a. The following scenario, lasting for 4 seconds, is set up. Laser trajectory is evenly rectilinear with constant velocity motion. The camera is fixed and it tracks a flat laser glint on a uniform background at the distance of 2 metres.
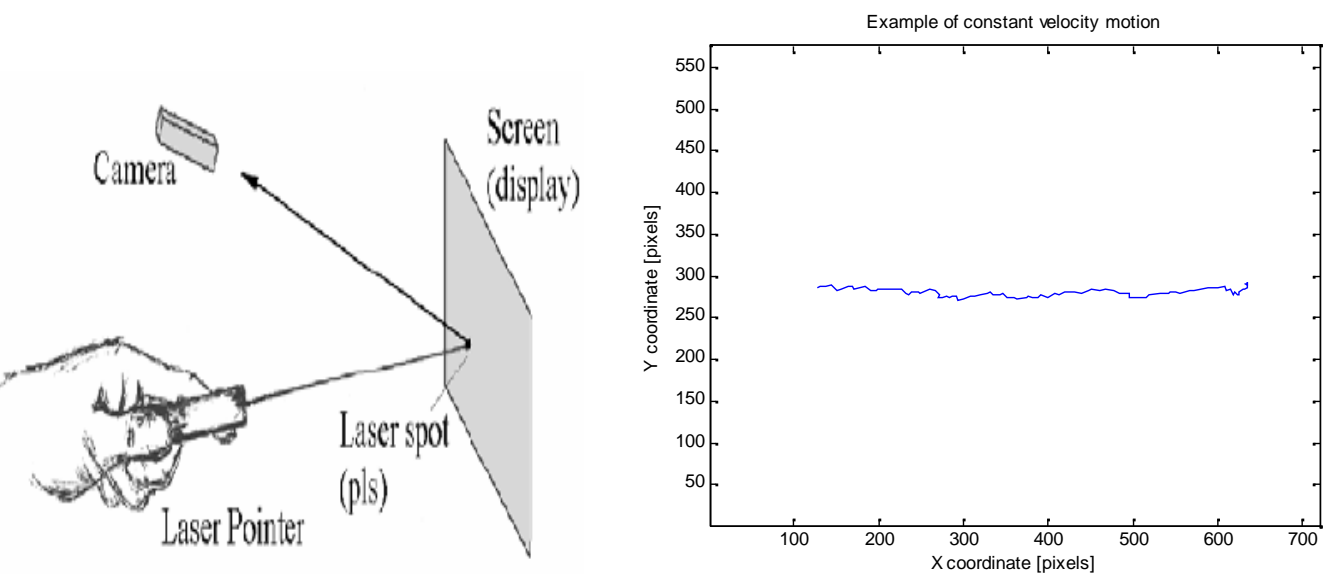

Figure (2): a) measurement, b) scenario

The target moved along x coordinate, whereas there are 295 pixels on y coordinate, figure 2.b. The determined variation along y coordinate can be said to be the very process noise. The variation of noise along y coordinate is 25 pixels $^{2}$, i.e. standard deviation is \pm 5 pixels. During the work process, it was noticed that the camera did not have constant selection period, i.e. constant time of frame formation of $40 \mathrm{~ms}$. Figure 3. shows discretization period on the basis of difference between processing time of two successively formed frames.

Depending on the obtained results, discretization period must have the variation of $0.14 \mathrm{~ms}$ and mathematical expection of $40 \mathrm{~ms}$. 


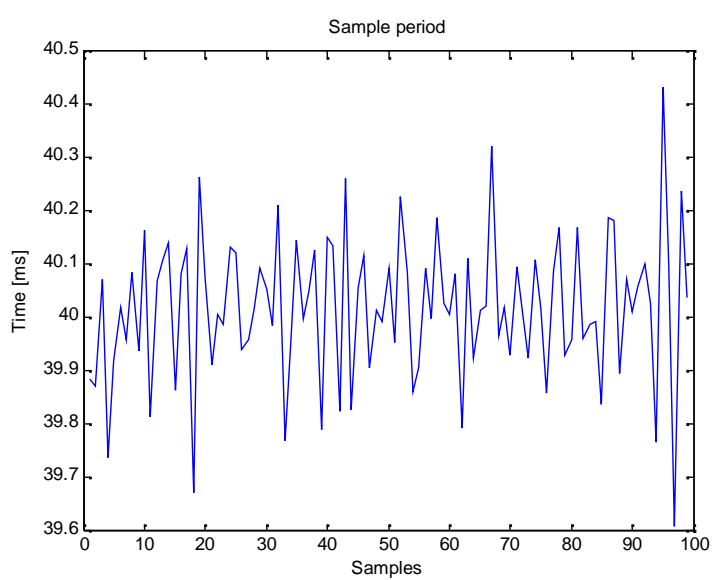

Figure (3): Discretization period between two successive frames

\section{Models description in filtration and prediction block:}

The influence of the model on the tracking quality will be analysed first. Here follows the description of models used in this paper. For all models $\mathrm{T}=0.04 \mathrm{~s}, \sigma^{2}=25$.

a) Constant velocity model is described as follows:

$$
F=\left[\begin{array}{ll}
1 & T \\
0 & 1
\end{array}\right], G=\left[\begin{array}{l}
0 \\
1
\end{array}\right], H=\left[\begin{array}{llll}
1 & 0 & 0 & 0 \\
0 & 0 & 1 & 0
\end{array}\right], Q=\left[\begin{array}{cc}
\sigma^{2} & 0 \\
0 & \sigma^{2}
\end{array}\right]
$$

b) Constant accelaration model is described as follows:

$$
\mathrm{F}=\left[\begin{array}{ccc}
1 & \mathrm{~T} & \frac{\mathrm{T}^{2}}{2} \\
0 & 1 & \mathrm{~T} \\
0 & 0 & 1
\end{array}\right], \mathrm{G}=\left[\begin{array}{l}
0 \\
0 \\
1
\end{array}\right], \mathrm{H}=\left[\begin{array}{cccccc}
1 & 0 & 0 & 0 & 0 & 0 \\
0 & 0 & 0 & 1 & 0 & 0
\end{array}\right], \mathrm{Q}=\left[\begin{array}{cc}
\sigma^{2} & 0 \\
0 & \sigma^{2}
\end{array}\right]
$$

c) Singer model is described in the following parameters:

maneuver time constant $\tau=0.4$, maximum target acceleration possible $a_{\max }=400$ pixels $/ \mathrm{s}^{2}$, maneuver probability with $a_{\max }, \mathrm{P}_{\max }=0.9$, probability that there will be no maneuver $\mathrm{P}_{0}=0.1$,

$$
\sigma_{a}^{2}=a_{\max }^{2} \frac{\left(1-4 P_{\max }-P_{o}\right)}{3}, \alpha=\frac{1}{\tau}
$$




$$
\mathrm{F}=\left[\begin{array}{ccc}
1 & \mathrm{~T} & \frac{\mathrm{T}^{2}}{2} \\
0 & 1 & \mathrm{~T} \\
0 & 0 & 1
\end{array}\right], \mathrm{GQG}^{\prime}=2 \sigma_{\mathrm{a}} \alpha\left[\begin{array}{ccc}
\frac{\mathrm{T}^{5}}{20} & \frac{\mathrm{T}^{4}}{8} & \frac{\mathrm{T}^{3}}{6} \\
\frac{\mathrm{T}^{4}}{8} & \frac{\mathrm{T}^{3}}{3} & \frac{\mathrm{T}^{2}}{2} \\
\frac{\mathrm{T}^{3}}{6} & \frac{\mathrm{T}^{2}}{2} & \mathrm{~T}
\end{array}\right] \mathrm{H}=\left[\begin{array}{cccccc}
1 & 0 & 0 & 0 & 0 & 0 \\
0 & 0 & 0 & 1 & 0 & 0
\end{array}\right]
$$

d) IMM algorithm

consists of two models, CV and CA described in equations (1) and (2). The probability of transfer from one onto the other model, process noise model and measurement noise model are defined as:

$\mu=\left[\begin{array}{cc}0.9 & 0.1 \\ 0.15 & 0.85\end{array}\right], \mathrm{Q}=\left[\begin{array}{cc}5^{2} & 0 \\ 0 & 5^{2}\end{array}\right], \mathrm{R}=\left[\begin{array}{ll}1 & 0 \\ 0 & 1\end{array}\right]$

\section{Sintetical trajectories:}

The first scenario is target motion with constant velocity of 150 pixels/s. Motion duration is $2.5 \mathrm{~s}$. Motion parameters are:

1. constant velocity lasting $0.5 \mathrm{~s}$,

2. exponential acceleration $a=\alpha \mathrm{e}^{\alpha \mathrm{t}}, \alpha=10$, lasting $0.5 \mathrm{~s}$,

3. uniform motion lasting $0.5 \mathrm{~s}$,

4. exponential deceleration $a=-\alpha \mathrm{e}^{\alpha \mathrm{t}}, \alpha=10$, last $0.5 \mathrm{~s}$ and

5. uniform motion lasting $0.5 \mathrm{~s}$.

Referent trajectory is presented in figure 4.a. Figure 4.b shows estimation and noisy trajectory with the variation of 25 pixels $^{2}$.
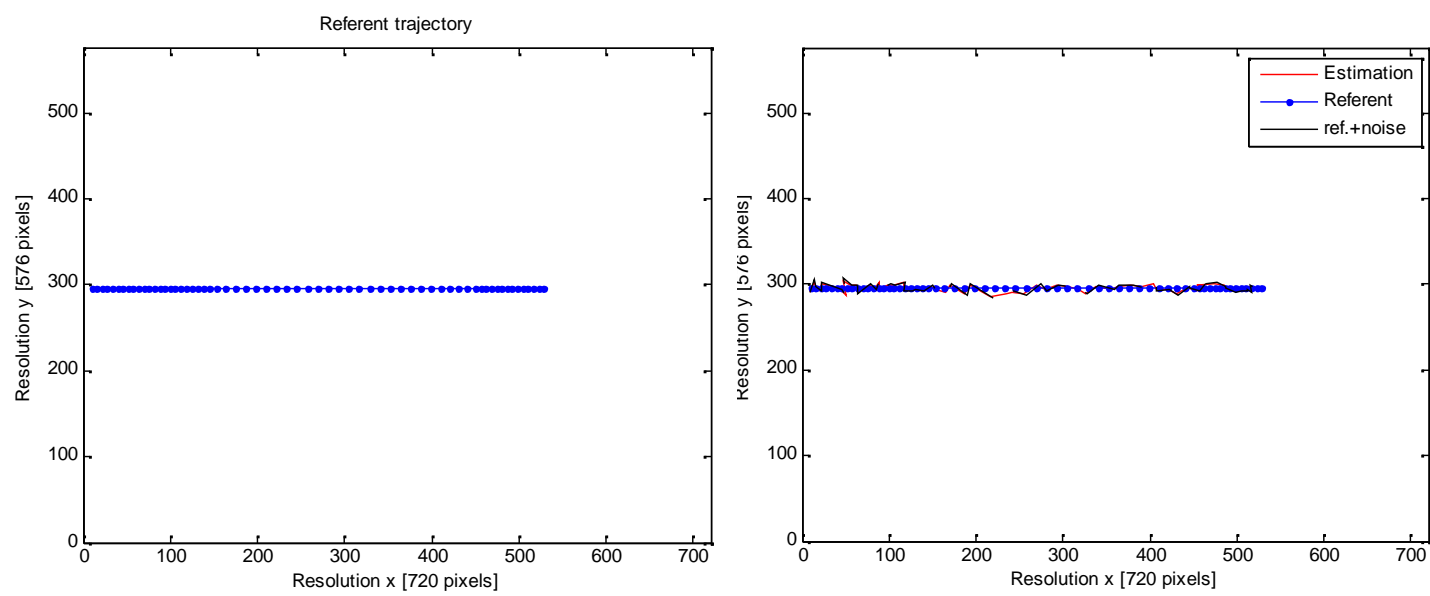

Figure (4): a) Referent trajectory, b) estimated trajectory 


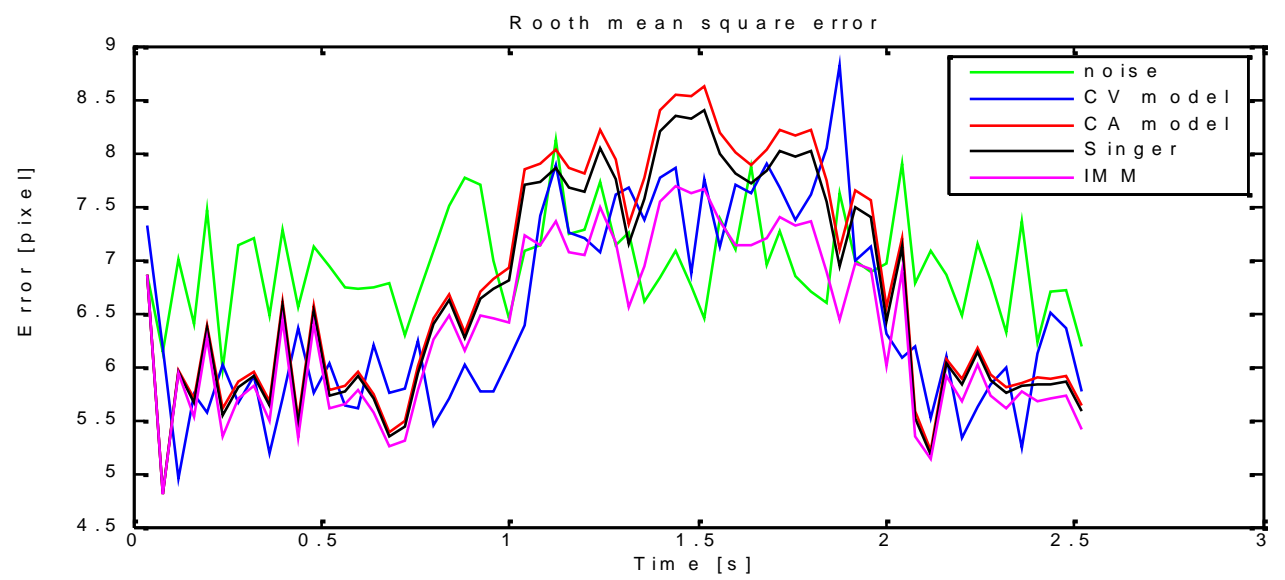

Figure (5): Position root mean squared error

Figure 5. represents root mean square error of position of all models implemented on referent trajectory in figure 4.a.

The second scenario is target motion with constant velocity of 150 pixels/s. Motion duration is $5 \mathrm{~s}$. Motion parameters are:

1. constant velocity lasting $1 \mathrm{~s}$,

2. left turn with the radial velocity of pi/5 rad/s lasting $1 \mathrm{~s}$,

3. uniform motion lasting $1 \mathrm{~s}$,

4. right turn with the radial velocity of $-\mathrm{pi} / 5 \mathrm{rad} / \mathrm{s}$ lasting $1 \mathrm{~s}$, and

5. uniform motion lasting $1 \mathrm{~s}$.

Referent trajectory is presented in figure 5.a. Figure 5.b shows estimation and noisy trajectory with the variation of 25 pixels $^{2}$.
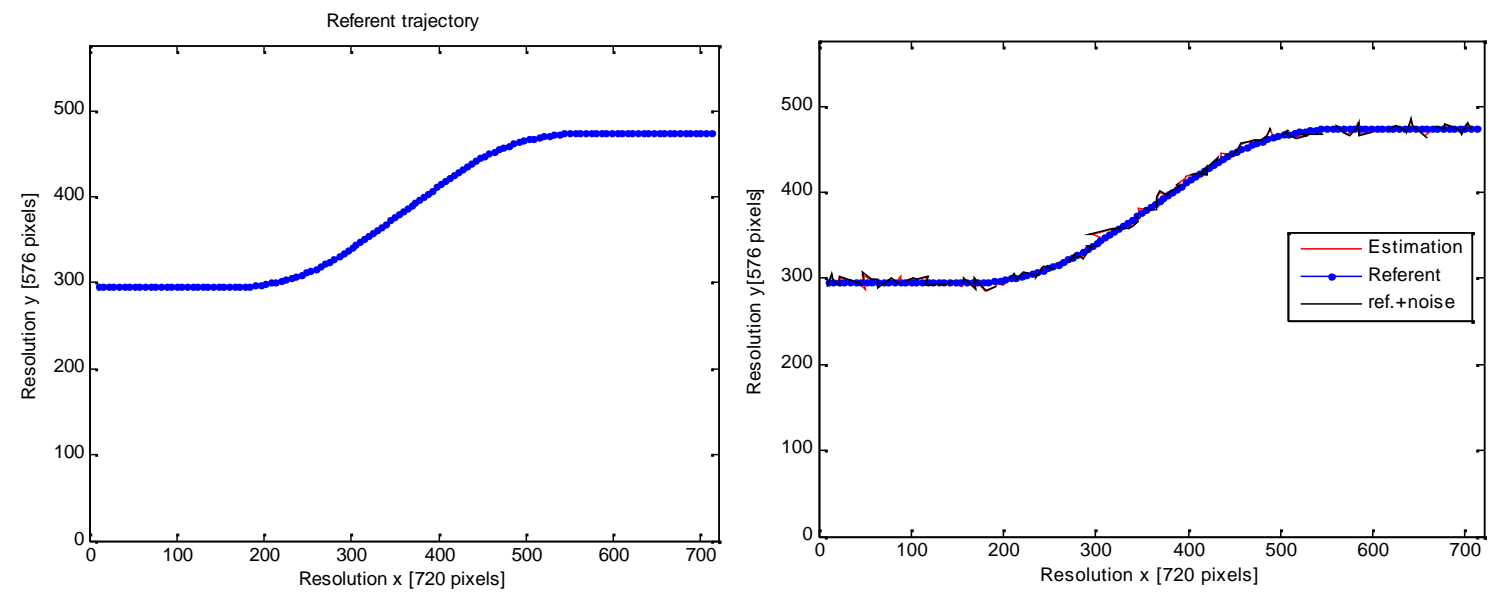

Figure (6): a) referent trajectory, b) estimated trajectory

Figure 7. represents root mean square error of position of all models implemented on referent trajectory in figure 6.a.

Here follows a characteristic case of the second scenario, when the target moves at constant velocity, and it maneuvers at the right angle. Target motion lasts for 2.5 seconds. Every $0.5 \mathrm{~s}$ there is a maneuver at $\mathrm{pi} / 2$. 


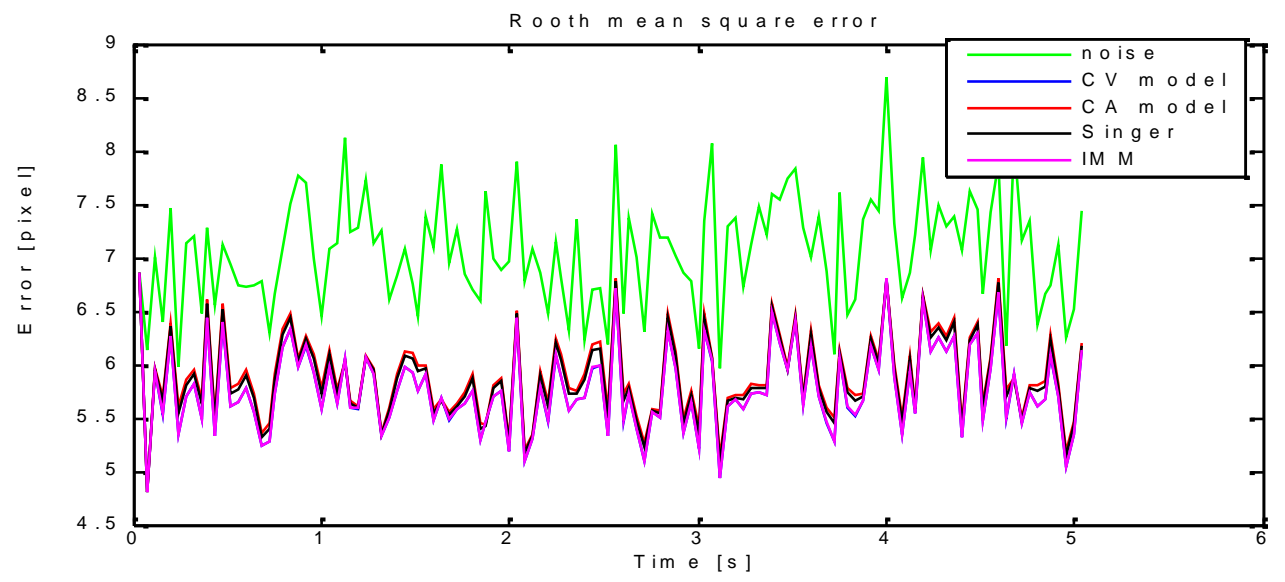

Figure (7): Position root mean squared error
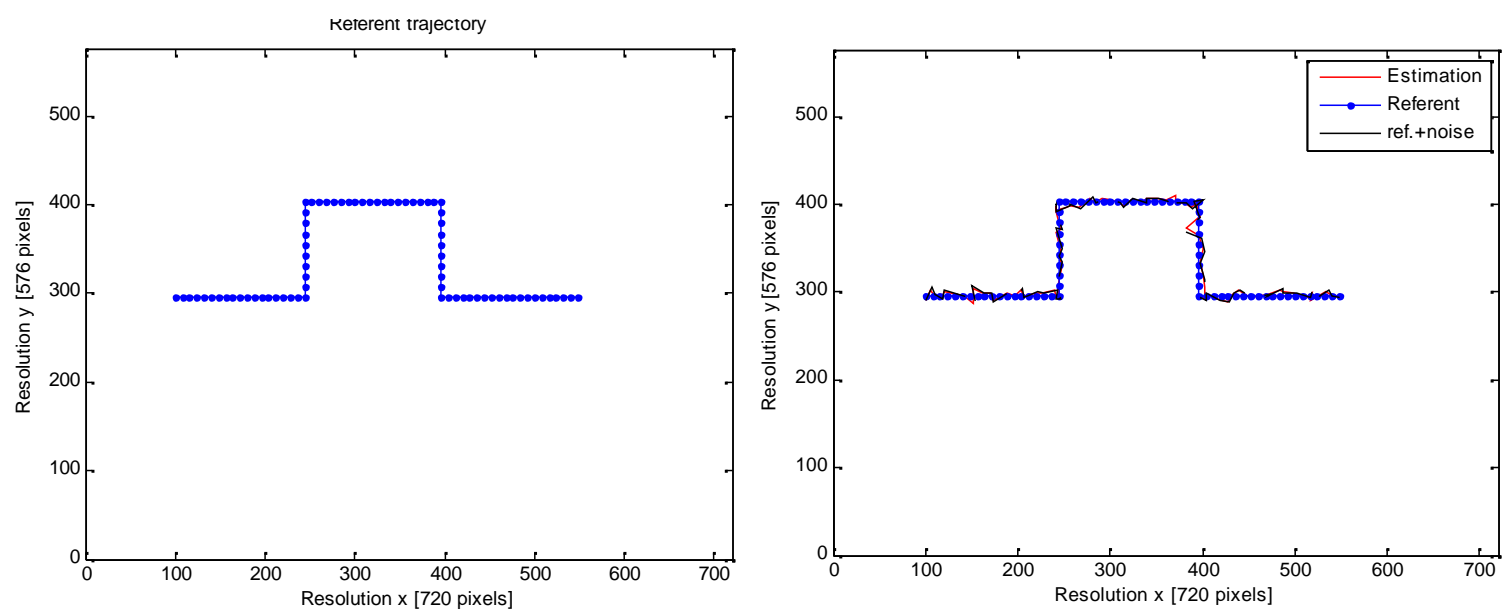

Figure (8): a) Referent trajectory, b) estimated trajectory

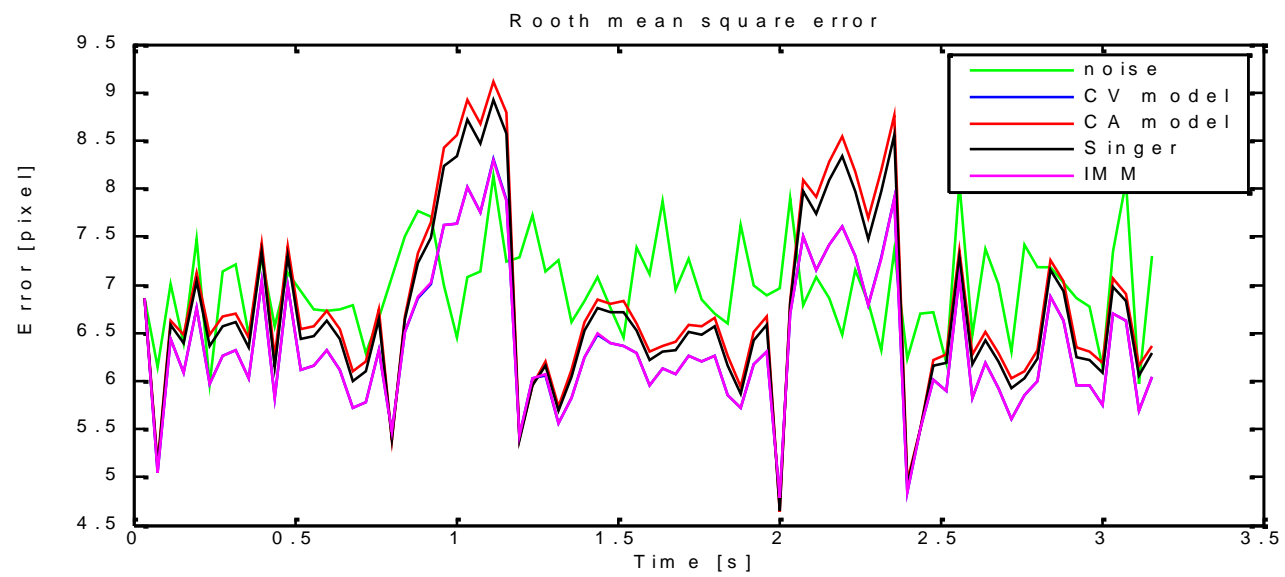

Figure (9): Position root mean squared error

On the basis of graphs 5,7 and 9, it can be concluded that IMM algorithm is best at tracking different target motions. As the task of tracking systems is to estimate the 
motion of targets with different parameters, it has been confirmed that models with constant values cannot be taken into consideration. In order to confirm the conclusion, another scenario was defined, and it will include all the mentioned motion parameters [13]. The target trajectory consists of 8 segments of 10 samples with sample period $\mathrm{T}=40 \mathrm{~ms}$. The target motion during each segment was:

1. uniform motion with constant velocity of $400 \mathrm{pixel} / \mathrm{s}$,

2. exponential acceleration motion, with acceleration $\mathrm{a}=a \mathrm{e}^{a t}, t$ denotes time since the segment start, and $a=2$,

3. exponential deceleration, with acceleration $\mathrm{a}=-a \mathrm{e}^{a t}, t$ denotes time since the segment start and $a=2$

4. right turn with the radial velocity of - pi $/ 0.35 \mathrm{rad} / \mathrm{s}$,

5. exponential acceleration with $\mathrm{a}=a \mathrm{e}^{a t}, a=2$,

6. exponential deceleration with $\mathrm{a}=a \mathrm{e}^{a t}, a=-2$,

7. left turn with radial velocity $\mathrm{pi} / 0.35 \mathrm{rad} / \mathrm{s}$, and

8. uniform motion.
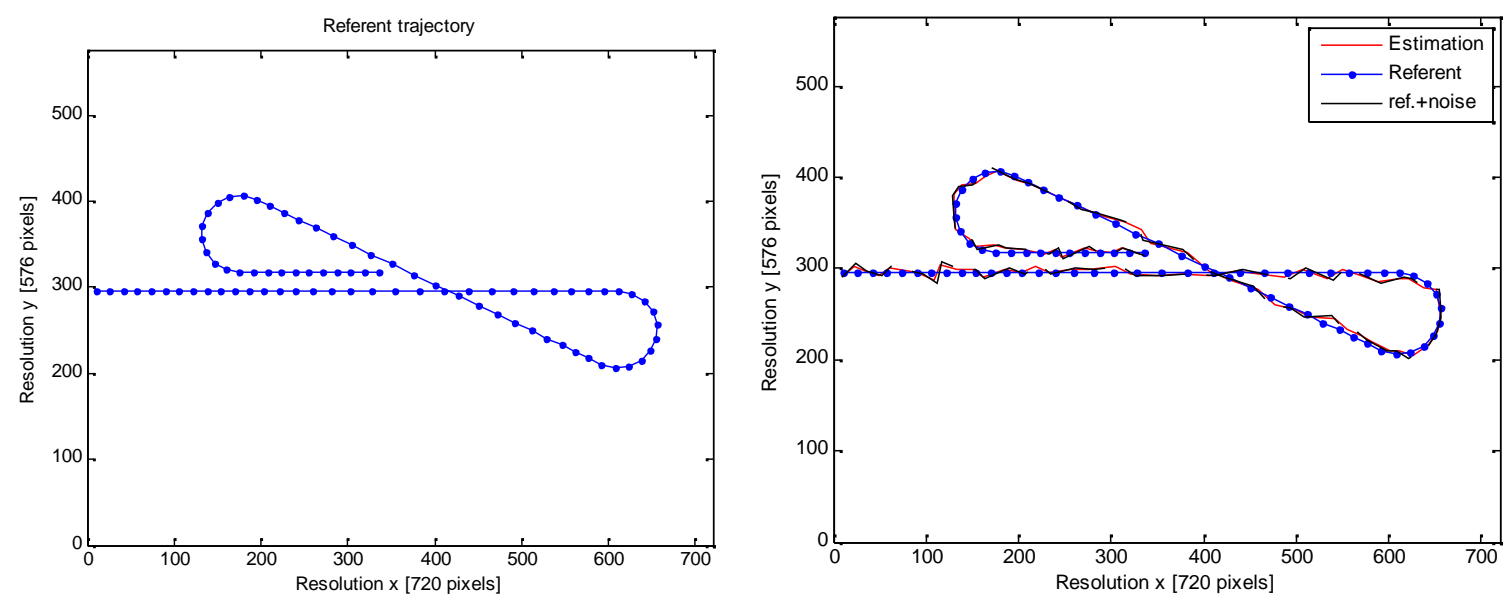

Figure (10): a) Referent trajectory, b) estimated trajectory

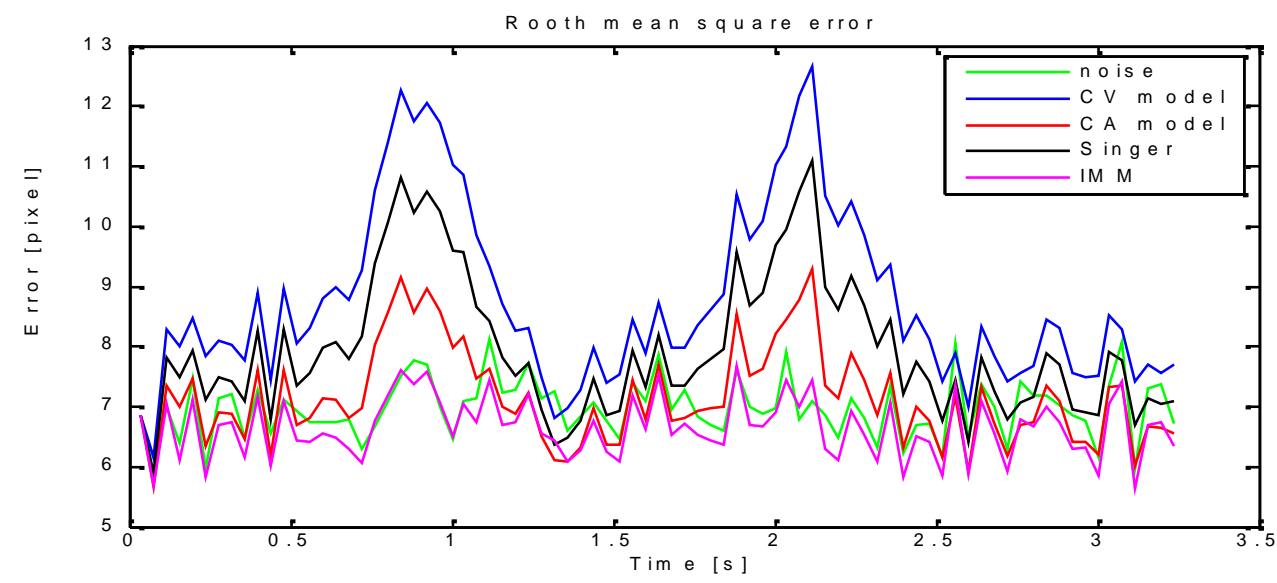

Figure (11): Position root mean squared error 
Finally, the conclusion that IMM algorithm is by far the best in comparison to all the processed filtration and prediction models, especially considering the measurement noise, has been confirmed. Now the models will be briefly implemented in the recorded video sequences.

\section{Recorded video sequences:}

The next scenario is the analysis of filtration and prediction model, but now based on the recorded sequences with red and infrared lasers. Both lasers are fixed, so that they give two laser glints, but with minimum difference in position. Video sequence is obtained by implementing infrared filter. Infrared video sequence is obtained by implementing the filter which does not let the light of less than $800 \mathrm{~nm}$ through. Video sequences are shown in figures 12.a and 12.b, and every fifth laser glint is represented. The width of the field of vision is set up at maximum.
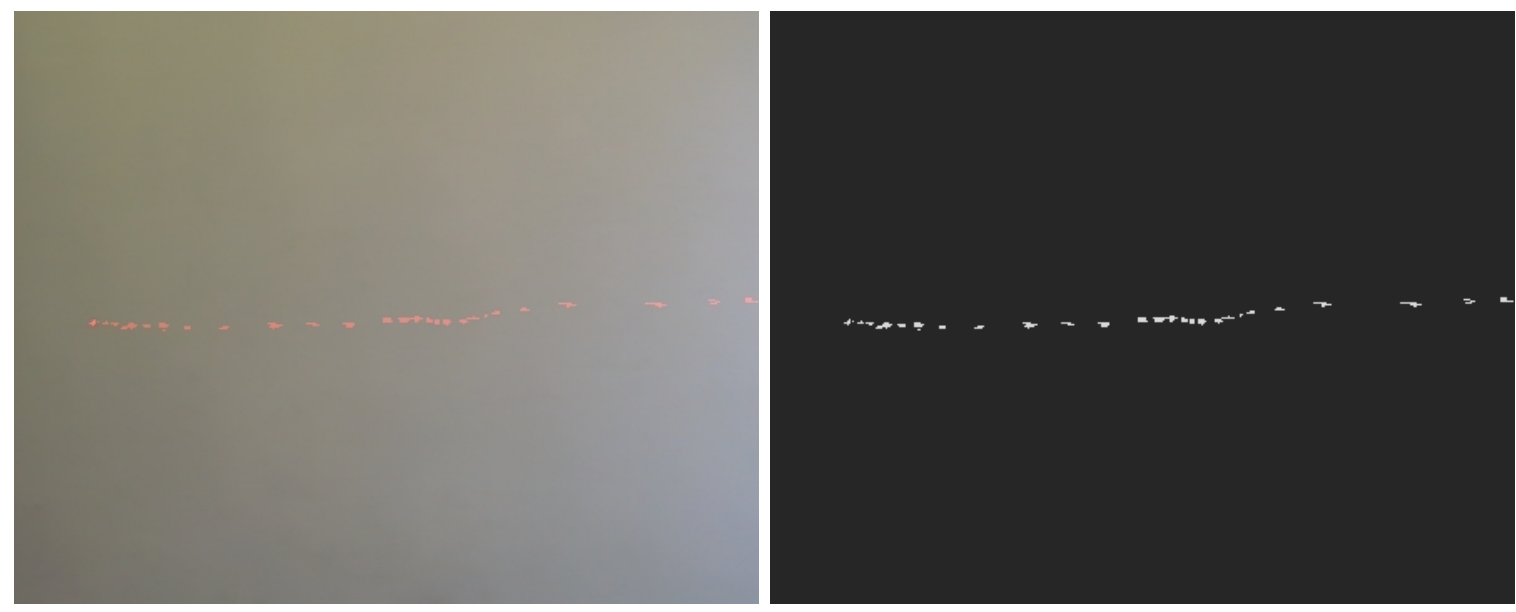

Figure (12): a) Recorded video sequence in visible spectrum, b) Recorded video sequence in infrared spectrum

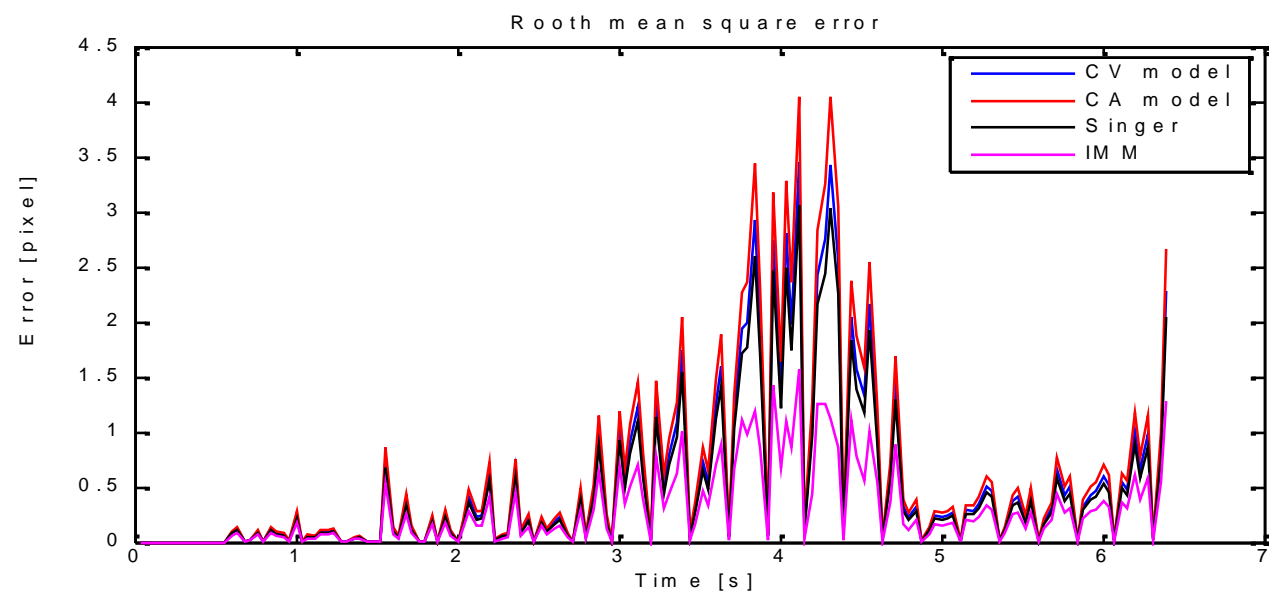

Figure (13): Position root mean squared error 

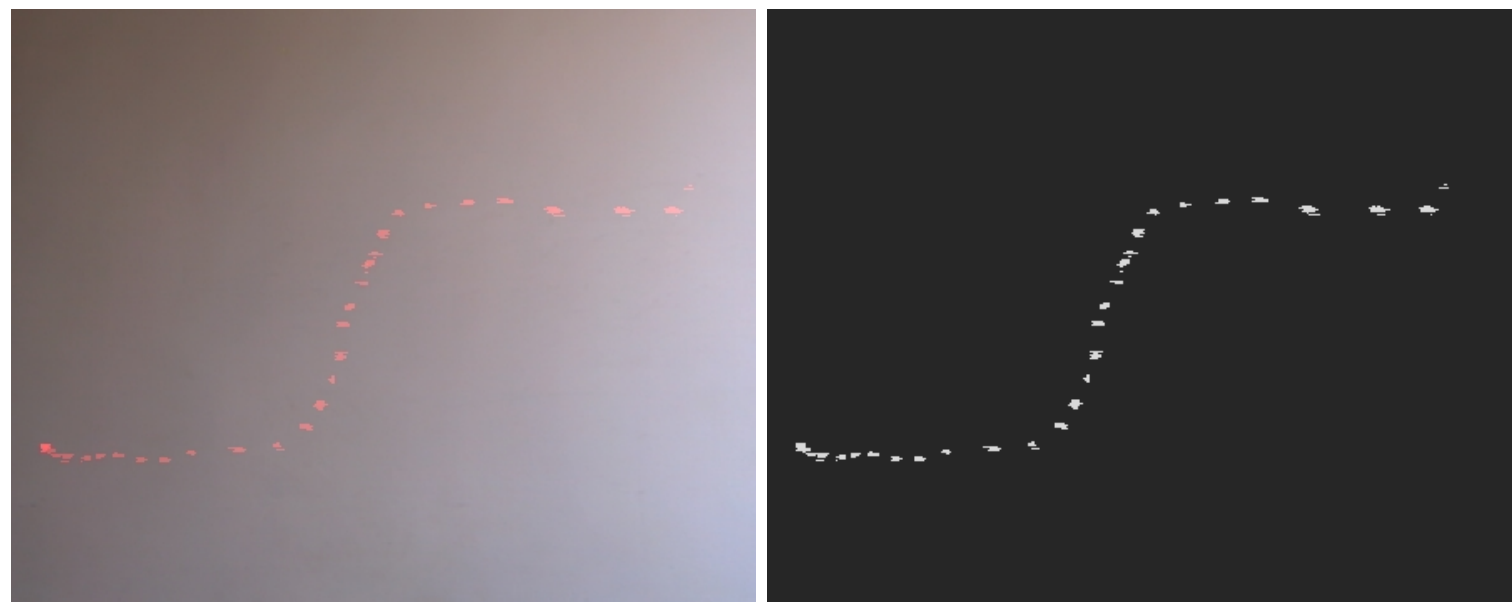

Figure (14): a) Recorded video sequence in visible spectrum, b) Recorded video sequence in infrared spectrum

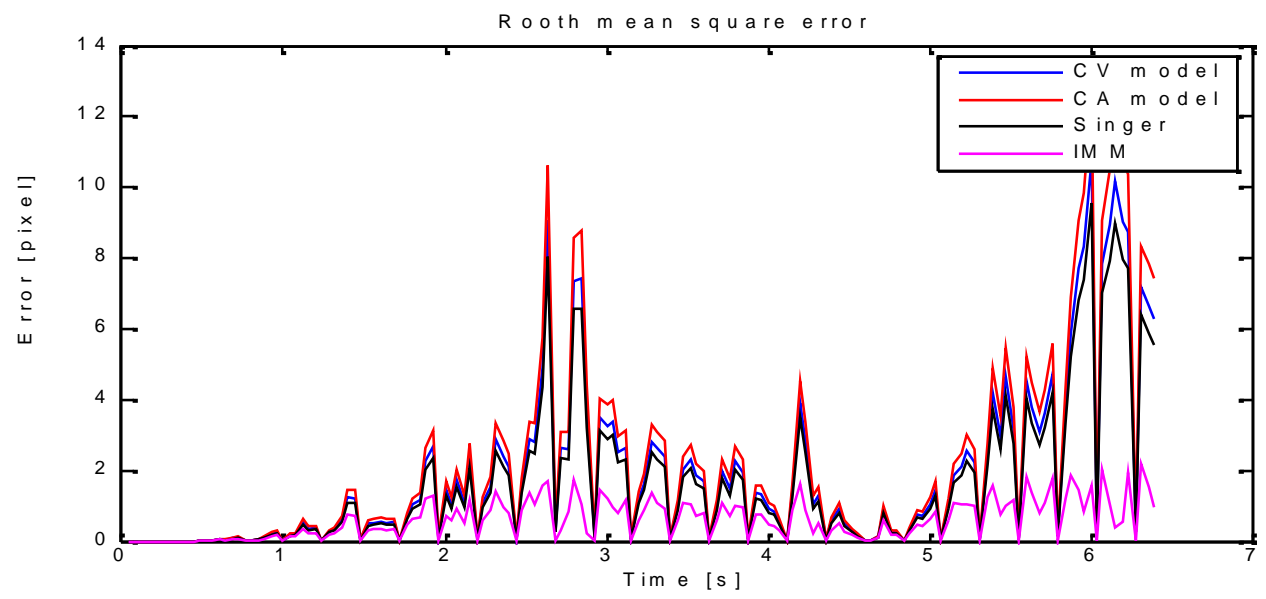

Figure (15): Position root mean squared error
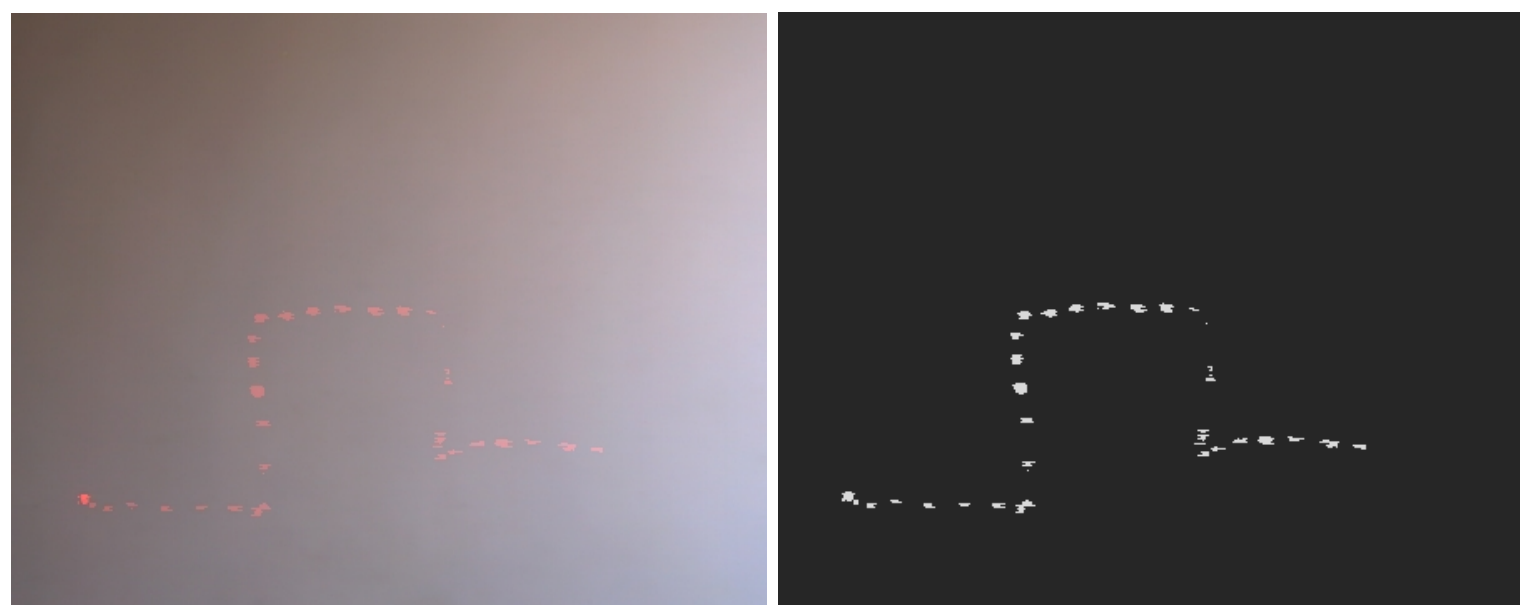

Figure (16) a) Recorded video sequence in visible spectrum, b) Recorded video sequence in infrared spectrum 


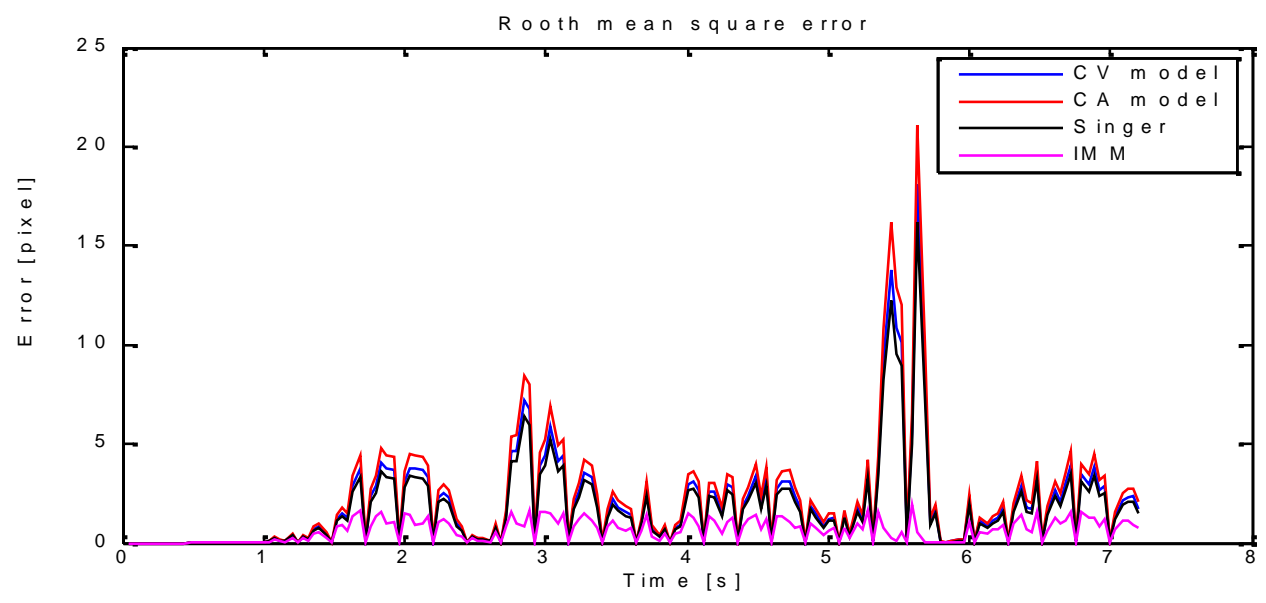

Figure (17): Position root mean squared error

IMM algorithm gives the best results. The difference in tracking quality is visible in all analysed trajectories. It can be seen that there are more errors in all algorithms, when recorded sequences are processed in relation to generated trajectories. No matter which spectrum is used for tracking, trajectory estimation is the same. Real scenario of target tracking is next, but with a controllable camera this time.

\section{Experiment result:}

Experiment results were obtained by tracking the laser glint whose parameters were described earlier and shown in figure 10.a. The camera is controlled by setting the angle that requires pan and tilt motion. IMM algorithm is used to estimate target tracking coordinates. Pan and tilt angles are then determined by zoom status and the distance of target coordinates from the centre of sensor field of vision. The information is then converted into a hexadecimal form and via serial communication sent to the camera. In this scenario, the discretization period is $\mathrm{T}=0.2 \mathrm{~s}$. Figure 18 . shows target trajectory and estimation. It can be seen that the camera was the first to detect the target. Next, it can be seen that target estimation is around the field of vision centre. The duration of tracking is $100 \mathrm{~s}$.
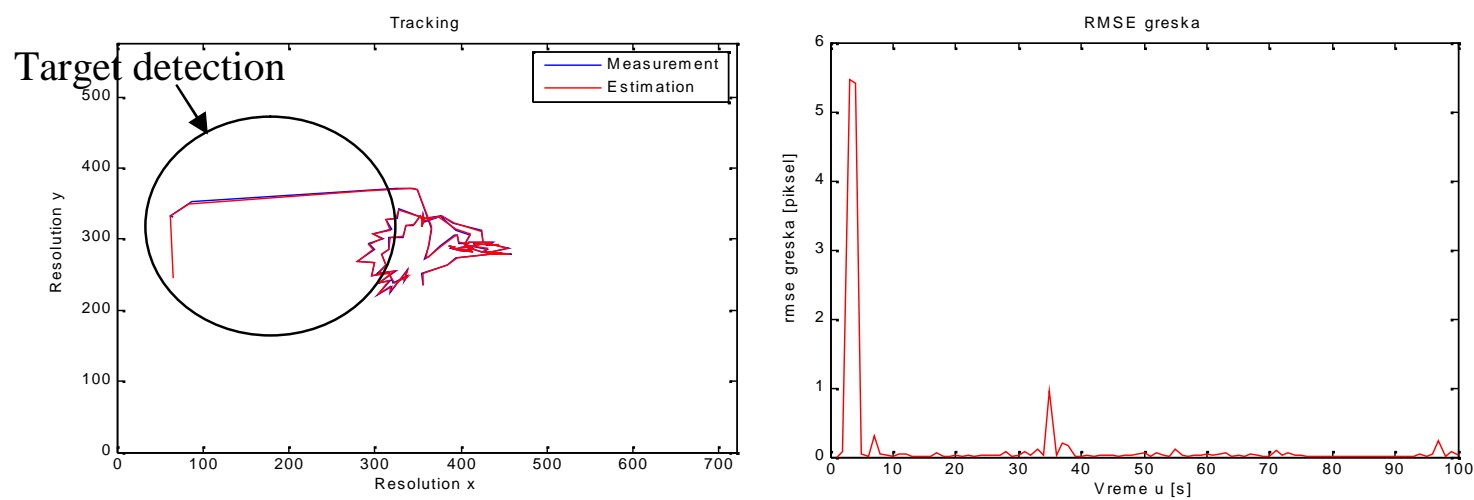

Figure (18): Target trajectory and estimation 

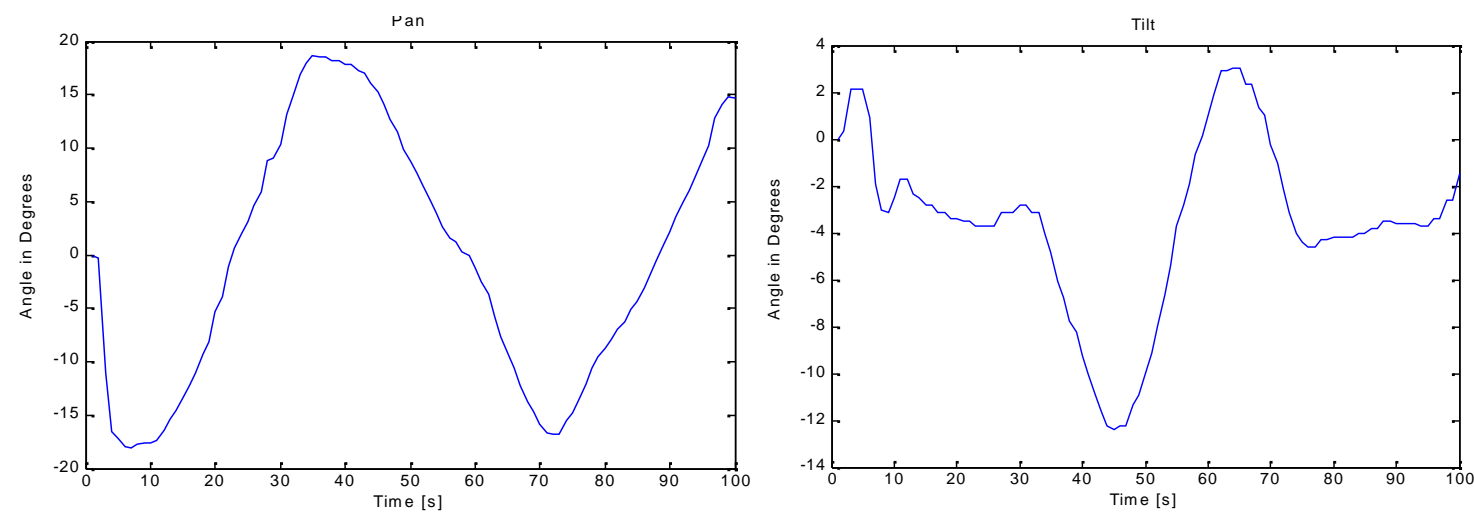

Figure (19): a) Camera azymuth, b) camera elevation

Figures 19.a and 19. b shows the angles of camera pan and tilt. The angles help determine the target motion.

\section{Conclusion:}

$\mathrm{CV}$, CA, Singer model and IMM algorithm within filtration and prediction blocks of target tracking systems were analysed in the paper. Target tracking quality has been analysed on the basis of generated characteristic trajectories and recorded video sequences. Laser glint has been monitored in visible and infrared spectrum. The results have been represented according to the criterion of position root mean squared error. IMM algorithm gave the best results of all the models processed.

An experiment of target tracking with controllable camera has been performed. IMM algorithm system had minimum position error. This paper can suggest target tracking system under different circumstances of daylight. Image sensor is VC-C50i camera which can with light wavelength filters track a target in different spectrums. Target trajectories were estimated with minimum position error using IMM algorithm in filtration and prediction block.

\section{Acknowledgment:}

Results presented in this paper are made within project No 47029 financed by Ministry of science and technology development Republic of Serbia. 


\section{References:}

[1] David Dwyer, Steve Hogg, Lee Wren, Gordon Cain, "Improved laser ranging using video tracking”, Proceedings of SPIE, Vol. SPIE-5082, pp. 21-29, 2003.

[2] Drigers R. G., Cox P. and Edwards T., " Introduction to Optoelectronics systems". Artech House, New York 1999.

[3] Sergey Matveyev, Konstantin Matveyev, "Tracking of a Laser Pointer Space Orientation by Using Single Camera", ICAT 2004.

[4] Karl Granstr"om, Christian Lundquist, Umut Orguner, "Tracking Rectangular and Elliptical Extended Targets Using Laser Measurements", Proceedings of the International Conference on Information Fusion, pp. 592-599, Chicago, IL, USA, Jul 2011.

[5] X.R. Chen, P. Cai, Wen Ku Shi, "An Application of the Kalman Filter in a Laser Tracking Measurement System", Journal Key Engineering Materials (Volumes 295 - 296), 2005, Volume Measurement Technology and Intelligent Instruments VI, Pages 245-252.

[6] Lewantowicz, Zdzislaw H., "Laser Pointing and Tracking Using an Adaptive Extended Kalman Filter", decembar 1976,

[7] Lokukaluge P. Perera, Carlos Guedes Soares, "Laser Measurement System based maneuvering Target tracking formulated by Adaptive Competitive Neural Networks", The Second International Conference on Adaptive and SelfAdaptive Systems and Applications, Adaptive 2010.

[8] Dillow, J. D.; Lewantowicz, Z. H.; Potter, R. M., "Laser glint tracking using an extended Kalman filter", NAECON '77; Proceedings of the National Aerospace and Electronics Conference, Dayton, Ohio, May 17-19, 1977. (A78-15551 0433) New York, Institute of Electrical and Electronics Engineers, Inc., 1977, p. 355-360.

[9] T.Kanade L.Matthies, R.Szeliski., "Kalman filter based algorithms for estimating depth from image sequences". International Journal of computer vision, 1989.

[10] P.Perona S. Soatto, R.Frezza. "Motion estimation via dynamic vision". In Proc. of the $33^{\text {rd }}$ IEEE Conf. on Decision and Control, CDC 94, 1994.

[11] R.Chellappa T.Broida. "Estimation of object motion parameters from noisy images". In IEEE trans. PAMI, Jan 1986.

[12] Srđan T. Mitrović, Goran D. Dikić, Željko M. Đurović, “ Hybrid Approach to Passive Ranging with Two Infrared Non-Imaging Senzors", $14^{\text {th }}$ International Conference on Aerospace Sciences and Aviation Technology - ASAT, Vol. 14, Cairo, Egypt, 2011, pp 046SP 1-12.

[13] D. Mušicki, B. F. La Scala, R. J. Evans, "Integrated Track Splitting Filter for Manoeuvring Targets", International Conference on Information Fusion, Fusion 2004, Stocholm, Sweden, July 2004. 